\title{
Pressure spectra for vortex models of fine-scale homogeneous turbulence
}

\author{
D. I. Pullin \\ Graduate Aeronautical Laboratories 105-50, California Institute of Technology, Pasadena, California 91125
}

(Received 5 July 1994; accepted 1 November 1994)

\begin{abstract}
Pressure spectra at large wave numbers are calculated for Lundgren-Townsend vortex models of the fine scales of homogeneous turbulence. Specific results are given for the Burgers vortex and also for the Lundgren-strained spiral vortex. For the latter case, it is found that the contribution to the shell-summed spectrum produced by the interaction between the axisymmetric and nonaxisymmetric components of the velocity field is proportional to $k^{-7 / 3}(k=|\mathbf{k}|$ is the modulus of the wave number) in agreement with Kolmogorov-type dimensional arguments. Numerical estimates of the dimensionless prefactors for this component are obtained in Kolmogorov scaling variables and comparisons are made with results from the Batchelor-Kolmogorov theory, and with experimental measurement. (C) 1995 American Institute of Physics.
\end{abstract}

\section{INTRODUCTION}

It has long been known that the dominant contribution to the volume or ensemble average of the mean-square pressure in a turbulent flow comes from the energy containing eddies. ${ }^{1-5}$ The value of the mean-square pressure gradient, on the other hand, appears to depend on contributions to the pressure correlation from wave numbers within the inertial subrange and viscous dissipation range of the velocity spectrum (Batchelor ${ }^{6,7}$ ). Whilst there have been several attempts to calculate the form of the pressure spectrum for the large scales of shear flow turbulence (e.g., Kraichnan, ${ }^{8}$ George et $a l^{9}{ }^{9}$ ), and while it is known that the pressure spectrum for isotropic turbulence is proportional to a certain two-point fourth-order velocity correlation, ${ }^{5,9}$ there does not yet appcar to have been an attempt to calculate the inertial-range pressure spectrum directly from a model of the velocity fielld

A prediction for the form of the spectrum in the inertial range of isotropic turbulence can be obtained by dimensional analysis based on Kolmogorov-type arguments; see (4) below. The scaling constant can be estimated ${ }^{9}$ in terms of the Kolmogorov prefactor for the velocity spectrum using a result due to Batchelor. ${ }^{6,7}$ Using the Millionshchikov ${ }^{10}$ jointnormal hypothesis for the probability distribution of the velocity field at two points, Batchelor uses dynamical arguments to obtain a relationship between the shell-summed pressure spectrum $\mathscr{G}(k)$ and the contracted velocity (energy) spectrum $E(k)$

$$
\mathscr{F}(k)=\frac{k^{2}}{2 \pi} \iiint E\left(k^{\prime}\right) E\left(\left|\mathbf{k}-\mathbf{k}^{\prime}\right|\right) \frac{\sin ^{4} \theta}{\left|\mathbf{k}-\mathbf{k}^{\prime}\right|^{4}} d \mathbf{k}^{\prime},
$$

where $\theta$ is the angle between the wave number vectors $\mathbf{k}$ and $\mathbf{k}^{\prime}$. When the Kolmogorov form of the inertial-range form for $E(k)$

$$
E(k)=\mathscr{K}_{0} \epsilon^{2 / 3} k^{-5 / 3},
$$

where $\overline{\boldsymbol{\epsilon}}$ is the average dissipation and $\mathscr{K}_{0}$ is the Kolmogorov prefactor, is used in (1), it is found that

$$
\begin{aligned}
\mathscr{F}(k) & =\mathscr{K}_{0}^{2} \bar{\epsilon}^{4 / 3} k^{-7 / 3} \int_{0}^{\pi} \int_{0}^{\infty} \frac{\eta^{1 / 3} \sin ^{5} \theta}{\left(\eta^{2}-2 \eta \cos \theta+1\right)^{17 / 6}} d \theta d \eta, \\
& =\frac{4536\left(\Gamma\left[\frac{4}{3}\right]\right)^{2}}{3025 \Gamma\left[\frac{5}{3}\right]} \mathscr{K}_{0}^{2} \bar{\epsilon}^{4 / 3} k^{-7 / 3},
\end{aligned}
$$

where the double integral has been evaluated by first expressing the integration with respect to $\eta$ in terms of associated Legendre functions, which then allows the $\theta$ integration to be performed explicitly. The coefficient has the numerical value 1.3245105 giving

$$
\mathscr{F}(k)=\mathscr{K}_{p} \bar{\epsilon}^{4 / 3} k^{-7 / 3}, \quad \mathscr{K}_{p} \approx 1.325 \mathscr{K}_{0}^{2} .
$$

This is in good agreement with Eq. (7.11) of Ref. 9 for which the integral in (1) was evaluated numerically using a model of $E(k)$ which agreed with (2) in the inertial range. Equation (4) is the Kolmogorov form of $\mathscr{G}(k)$ with the prefactor $\mathscr{G}_{p}$ related to $\mathscr{K}_{0}$ using (1). We comment that equations (22.28)-(22.30) of Monin and Yaglom ${ }^{5}$ give relations, equivalent to (1)-(4), between the second-order velocity and pressure structure functions.

In the present paper we calculate directly the one-time two-point pressure correlation in homogeneous turbulence consisting of a randomly oriented collection of vortex structures of the Lundgren ${ }^{11-13}$ (Ref. 11 is henceforth referred to as L)-Townsend ${ }^{14}$ type. Our aim is to elucidate a means of relating the shell-summed pressure correlation to a velocity field inside vortex structures which satisfies local dynamics. In Sec. II the method of calculating the pressure spectrum from the pressure field inside the vortex is given. The kinematics closely follow those given by L for the energy spectrum with the difference that the contraction of the vorticity correlation function is here replaced by the pressure correlation. A strong assumption is introduced, namely that where individual vortices are subject to a strain field that stretches vortex lines, correlations involving the pressure field of the straining motions are associated with large scales, which, it is supposed, is producing the strain. Since these scales lie outside the range of the present model, the contributions produced by them to the calculated pressure spectrum are omitted from consideration. In Sec. III the pressure spectrum pro- 
duced by an ensemble of Burgers ${ }^{15}$ vortices is calculated and is found to contain no inertial range. The stretched-spiral vortex is studied in Sec. IV. The pressure field is first estimated from the radial momentum equation combined with a truncation of an expansion of the velocity field in inverse powers of a stretched time scale. The resulting power spectrum of the pressure contains two components, one associated with the axisymmetric or azimuthally averaged pressure, and the second arising from an interaction between the axisymmetric component of the azimuthal velocity and the spiral, or nonaxisymmetric velocity modes. This latter component is found to exhibit a $k^{-7 / 3}$ decay at large wave numbers in agreement with the exponent in (4), independent of the specific details of the spiral structure. For the special realization of the spiral structure studied by Pullin and Saffman, ${ }^{16}$ namely a thin shear layer in the process of rollup, numerical estimates of the prefactor $\mathscr{K}_{p}$ are made. A comparison of the one-dimensional pressure spectrum with the measurements of George et al. ${ }^{9}$ is also discussed.

\section{THE PRESSURE SPECTRUM}

We consider homogeneous anisotropic turbulence in an incompressible fluid with constant density $\tilde{\rho}$. The pressure at a point $\mathbf{x}$ is $p(\mathbf{x}, t)$. We define the pressure correlation $P(\mathbf{r})$ by

$$
P(\mathbf{r})=\frac{1}{\overline{\boldsymbol{\rho}}^{2}} \overline{\overline{p(\mathbf{x}) p(\mathbf{x}+\mathbf{r})}},
$$

where $\mathbf{r}$ is the separation and the double overbar denotes a probability or ensemble average for the field of motion. The pressure spectrum function $\Pi(\mathbf{k})$ is defined as

$$
\Pi \mathbf{I}(\mathbf{k})=\frac{1}{8 \pi^{3}} \iiint P(\mathbf{r}) e^{-i \mathbf{k} \cdot \mathbf{r}} d \mathbf{r},
$$

and the shell-summed pressure spectrum is

$$
\mathscr{g}(k)=\iint_{S(\mathbf{k})} \Pi(\mathbf{k}) d S(\mathbf{k}) \text {, }
$$

where $S(\mathbf{k})$ is the surface of a sphere in $\mathbf{k}$ space of radius $k$.

We now assume that the turbulence consists of a large collection of straight vortex structures. Each structure has the property that the vorticity is aligned with the structure axis, and that the vorticity magnitude does not depend on the axial coordinate. We refer to vorticity distributions like this as $c y$ lindrically unidirectional. ${ }^{17}$ In the spirit of these LundgrenTownsend models, it is assumed that individual vortices may be stretched, rotated or tilted by the velocity-gradient field of larger-scale structures, and that internally the vorticity is evolving according to local Navier-Stokes or Euler dynamics which may respond to the external field. The vortices themselves, however, are not coupled dynamically, and in this sense the turbulence properties that can be calculated from a collection of such structures must be thought of arising from a random superposition of deterministic but independent velocity fields. Lundgren ${ }^{11}$ showed that a statistically homogeneous collection of strained-spiral vortices with cylindrically unidirectional vorticity has a $k^{-5 / 3}$ inertial range for the velocity spectrum and a $k^{-1}$ spectral range for the mixing of a passive scalar. ${ }^{12}$ Pullin and Saffman ${ }^{16}$ have used this model to calculate the higher-order statistics of the velocity-gradient field and the dissipation spectrum. ${ }^{18}$ Evidence that structures of the cylindrically unidirectional type are characteristic of the small scales of isotropic turbulence has been provided by several numerical simulations (see Refs. 19-21).

We denote vortex-fixed axes by $\left(x_{1}, x_{2}, x_{3}\right)$ with $x_{3}$ in the direction of the vorticity. Pullin and Saffman's ${ }^{16}$ (PS) equation (B16) show that for this model the power spectrum of the product of two arbitrary scalar functions of position in a vortex cross section $F_{1}\left(x_{1}, x_{2}\right)$ and $F_{2}\left(x_{1}, x_{2}\right)$ can be expressed in terms of the product of the sectional Fourier transforms of $F_{1}$ and $F_{2}$. We utilize their Eq. (B16) choosing here $F_{1}=F_{2}=P\left(x_{1}, x_{2}, t\right) \equiv p\left(x_{1}, x_{2}, t\right) / \tilde{\rho}$. The dependence of pressure on only the $x_{1}, x_{2}$ coordinates within a vortex (plus time $t$ ) will be discussed subsequently. The shellsummed pressure spectrum is then given by

$$
\mathscr{G}(k)=\frac{4 \pi^{2} k}{L^{3}} \sum_{w} l_{w} \int_{0}^{2 \pi}\left|\mathscr{P}^{(w)}(k \cos \vartheta, k \sin \vartheta, t)\right|^{2} d \vartheta
$$

where

$$
\begin{aligned}
& \mathscr{P}^{(w)}\left(k_{1}, k_{2}, t\right)=\frac{1}{4 \pi^{2}} \int_{-\infty}^{\infty} \int_{-\infty}^{\infty} P^{(w)}\left(x_{1}, x_{2}, t\right) \\
& \times e^{-i k_{1} x_{1}-i k_{2} x_{2}} d x_{1} d x_{2}, \\
& P^{(w)}\left(x_{1}, x_{2}, t\right)=\int_{-\infty}^{\infty} \int_{-\infty}^{\infty} \not{g}(w)\left(k_{1}, k_{2}, t\right) \\
& \times e^{i k_{1} x_{1}+i k_{2} x_{2}} d k_{1} d k_{2} .
\end{aligned}
$$

In (8) and (9) the sum is over all vortices, denoted individually by the superscript $(w)$, which fill a large box of volume $L^{3}$ and $l^{(w)}(t)$ is the instantaneous length of a structure. In obtaining (8) and (9) it is assumed that although the vortices are to be thought of as of finite length, end effects, where vortex lines may diverge or become kinked where they reconnect to other vortex structures, do not contribute substantially to $P(\mathbf{r})$. Results like (8) for the power spectra of scalar quantities are valid if the turbulence is homogeneous but anisotropic in the sense of a nonuniform distribution of the orientations of individual members of the vortex ensemble relative to fixed laboratory axes. This is not the case when $F_{1}$ and $F_{2}$ are vector or tensor quantities, in which case integration over the probability distribution of the orientations will in general lead to results which differ for isotropic. and for anisotropic turbulence. ${ }^{17}$

An implicit assumption has been introduced concerning $P(\mathbf{r})$. If, in the context of the vortex model discussed above, individual vortices are being stretched or tilted by some external velocity gradient field, supposed to be produced by larger structures, then the pressure field produced inside the vortices will in general contain contributions from quadratic products of velocity components associated with this external field. We have assumed that contributions to the pressure correlations corresponding to (8) on scales comparable to the vortex cross-sectional diameter are provided only by the velocity field of the vortex itself, and that any correlations in- 
volving that part of the pressure field produced by the straining or tilting motions come from large scales with wave numbers sufficiently small so as to lie outside the range of the present model. Physically this means that pressure correlations within the structures themselves are related to the radial pressure gradients generated by swirling motions inside the vortex. Thus, in determining the pressure field contributing to (8), we will consider only velocity components $u_{1}, u_{2}$ in the directions $x_{1}, x_{2}$ respectively which are produced by the vortex. Hence $P$ appearing in (8) and (9) will depend only on these coordinates, and not on $x_{3}$. We remark that the effect of, for example, vortex stretching in the $x_{3}$ direction may still be present parametrically through any dependence of $u_{1}, u_{2}$ on the components of large-scale strain. This will become apparent in detailed calculations to be described later.

The integrations in (8) and in the first of (9) may be treated by utilizing the method developed in $L$ for calculating the energy spectrum. Briefly, when a transformation to polar coordinates is made with $x_{1}=r \cos \theta, x_{2}=r \sin \theta$, and $P(r, \theta, t)$ is expanded as a Fourier series

$$
P(r, \theta, t)=\sum_{n=-\infty}^{\infty} P_{n}(r, t) e^{i n \theta}, \quad P_{-n}=P_{n}^{*},
$$

the integrations with respect to the azimuthal angles $\theta$ and $\vartheta$ can be performed and it follows that the integral in (8) can be written as

$$
\begin{aligned}
& \int_{0}^{2 \pi}|\mathscr{P}|^{2} d \vartheta=\frac{1}{2 \pi}\left(I_{0} I_{0}^{*}+2 \sum_{n=1}^{\infty} I_{n} I_{n}^{*}\right), \\
& I_{n}(k, t)=\int_{0}^{\infty} J_{n}(k r) P_{n}(r, t) r d r
\end{aligned}
$$

where $*$ denotes the complex conjugate and $J_{n}$ is the Bessel function. Finally, the sum over vortices in $L^{3}$ at time $t$ is replaced by a time integration over the lifetime of an individual typical vortex (ergodic hypothesis), weighted by the rate $N_{c}$ at which vortex length is produced in the volume

$$
\sum_{w} \cdots \rightarrow N_{c} \int_{t_{1}}^{t_{2}} \cdots d t
$$

where $t_{1}$ and $t_{2}$ are upper and lower cutoff times, which in general will depend on the specific model. Substituting (11) and (13) into (8) gives

$$
\mathscr{F}(k)=\frac{2 \pi N_{c} k}{L^{3}} \int_{t_{1}}^{t_{2}} l(t)\left(I_{0} I_{0}^{*}+2 \sum_{n=1}^{\infty} I_{n} I_{n}^{*}\right) d t,
$$

and $l(t)$ now refers to the length of the vortex during its cvolution.

\section{THE BURGERS VORTEX}

In the case where the velocity field of the vortex is axisymmetric, only the $I_{0}$ integral in (11) is nonzero. From the $r$-momentum equation, the radial pressure gradient produced by the velocity field of the vortex is

$$
\frac{\partial P_{0}}{\partial r}=\frac{u_{\theta_{0}}^{2}(r, t)}{r},
$$

where $u_{\theta_{0}}(r, t)$ is the $\theta$ velocity. Integrating by parts and using the recurrence relation for the $J_{n}$ we can obtain

$$
\begin{aligned}
I_{0}(k, t)= & \int_{0}^{\infty} J_{0}(k r) P_{0}(r, t) r d r \\
= & -\frac{1}{2} \int_{0}^{\infty} J_{0}(k r) u_{\theta_{0}}^{2}(r, t) r d r \\
& -\frac{1}{2} \int_{0}^{\infty} J_{2}(k r) u_{\theta_{0}}^{2}(r, t) r d r .
\end{aligned}
$$

The Burgers vortex has a vorticity and $\theta$-velocity distribution produced by this vorticity given, respectively, by

$$
\begin{aligned}
& \omega(r)=\frac{\Gamma a}{4 \pi \nu} \exp \left(-\frac{a r^{2}}{4 \nu}\right), \\
& u_{\theta_{0}}(r)=\frac{\Gamma}{2 \pi r}\left[1-\exp \left(-\frac{a r^{2}}{4 \nu}\right)\right],
\end{aligned}
$$

where $\Gamma$ is the vortex circulation, $a$ is the strain rate in the direction of the vortex lines, and $\nu$ is the kinematic viscosity.

Using (18) the relevant integrations (16) can be performed and it is found that

$$
\begin{aligned}
I_{0}(k) & =-\frac{1}{2} L_{0}(k)-\frac{1}{2} L_{2}(k), \\
L_{0}(k) & \equiv \int_{0}^{\infty} J_{0}(k r) u_{\theta_{0}}^{2}(r) r d r \\
& =\frac{\Gamma^{2}}{8 \pi^{2}}\left[2 E_{1}\left(\frac{k^{2} \nu}{a}\right)-E_{1}\left(\frac{k^{2} \nu}{2 a}\right)\right], \\
L_{2}(k) & \equiv \int_{0}^{\infty} J_{2}(k r) u_{\theta_{0}}^{2}(r) r d r \\
& =\frac{\Gamma^{2} a}{4 \pi^{2} \nu k^{2}} \exp \left(-\frac{k^{2} \nu}{a}\right)\left[\exp \left(\frac{k^{2} \nu}{2 a}\right)-1\right],
\end{aligned}
$$

where $E_{1}[z]$ is the exponential integral (Abramowitz and Stegun, ${ }^{22}$ Eq. 5.1.1). When it is assumed that the vortex length is stretched as $l^{(w)}=l_{0} \exp (a t)$, where $l_{0}$ is the initial length, the time integral in (14) can be performed giving, for the pressure spectrum produced by a randomly oriented collection of Burgers vortices

$$
\mathscr{G}(k)=\frac{\pi N}{2 a} \exp \left(a t_{2}\right) k\left[L_{0}(k)+L_{2}(k)\right]^{2}
$$

where $N=N_{c} l_{0} / L^{3}$ and where we have put $t_{1}=0$. Since $N$, the rate of creation of vortex length per unit time per unit volume, has the dimensions length ${ }^{-2} \times$ time $^{-1}$, it may be verified that the right-hand side of $(21)$ has the required dimensions length ${ }^{5} \times$ time $^{-4}$. The $\exp \left(a t_{2}\right)$ prefactor occurs in (21) because of the exponential strctch of the vortex. Since our primary interest is in the form of $\mathscr{G}(k)$, no attempt has been made to estimate $t_{2}$, using, for example, a condition that the collection of Burgers vortices cannot overfill space. 


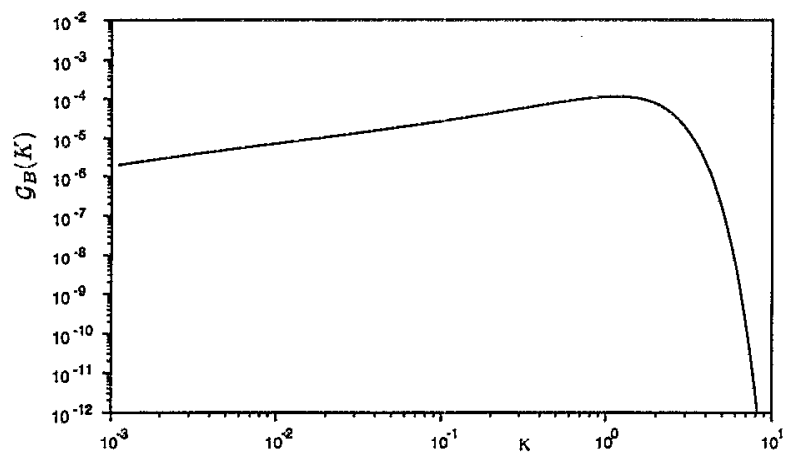

FIG. 1. Pressure spectrum plotted as $\mathscr{S}_{\mathrm{B}}(K)$ vs $K$ for a random collection of Burgers vortices.

The asymptotics of the exponential integral may be used to show that when $k \ll(a / \nu)^{1 / 2}$, to leading order

$$
\mathscr{F}(k) \sim k\left[\log \left(k^{2} \nu / a\right)\right]^{2} \text {. }
$$

The pressure spectrum, like the energy spectrum for Burgers vortices, contains no inertial range. Defining a dimensionless wave number $K=2 k(\nu / a)^{1 / 2}$ allows definition of a dimensionless function of $K$

$$
\mathscr{G}_{\mathrm{B}}(K)=\Gamma^{-4} K\left[L_{0}(K)+L_{2}(K)\right]^{2},
$$

which, by comparison with (21), may be used to depict the form of the variation of $\mathscr{S}$ over the full wave number range. This is shown in Fig. 1.

\section{THE STRETCHED-SPIRAL VORTEX}

\section{A. Vorticity and velocity fields}

We next consider the pressure spectrum produced by a collection of stretched spiral vortices. For the nonaxisymmetric motions we will confine attention to the inviscid spiral. Thus the present results are restricted to wave numbers lying between the range of dissipation and energy containing scales. The unsteady vorticity in the spiral is ${ }^{11}$

$$
\begin{aligned}
& \omega(r, \theta, t)=e^{a t} \hat{\omega}(\rho, \theta, \tau), \\
& \hat{\omega}(\rho, \theta, \tau)=\sum_{-\infty}^{\infty} \hat{\omega}_{n}(\rho, \tau) e^{i n \theta}, \\
& \hat{\omega}_{n}(\rho, \tau)=f_{n}(\rho) e^{-i n \Omega(\rho) \tau}, \quad n \neq 0, \\
& \hat{\omega}_{0}(\rho, \tau)=g(\rho, \tau)+f_{0}(\rho, \tau), \\
& \rho(r, t)=r e^{a t / 2}, \quad \tau(t)=\left(e^{a t}-1\right) / a,
\end{aligned}
$$

where

$$
\frac{1}{\rho} \frac{d}{d \rho}\left[\rho^{2} \Omega(\rho)\right]=g(\rho)+f_{0}(\rho) .
$$

In the above, " $a$ " is again the stain rate in the direction of the vortex lines, assumed to be provided by larger scales, $\rho$ now refers to a stretched spatial coordinate and $\tau$ a stretched time variable corresponding to a strictly two-dimensional evolution, $e^{a t} \cdot f_{0}(\rho)$ is the $\theta$-averaged vorticity for the spiral and $e^{a t} g(\rho)$ specifies some axisymmetric background vortic- ity field. The functions $f_{n}(\rho)$ define the structure of the spiral and at this stage their dependence on $n$ remains unspecified.

The required velocity components can be calculated from an equivalent two-dimensional streamfunction $\hat{\psi}(\rho, \theta, \tau)$ as

$$
\begin{aligned}
& u_{r}(r, \theta, t)=-\frac{1}{2} a r+e^{a t / 2} \hat{u}_{\rho}(\rho, \theta, \tau), \quad \hat{u}_{\rho}=\frac{1}{\rho} \frac{\partial \hat{\psi}}{\partial \theta}, \\
& u_{\theta}(r, \theta, t)=e^{a t / 2} \hat{u}_{\theta}(\rho, \tau), \quad \hat{u}_{\theta}=-\frac{\partial \hat{\psi}}{\partial \rho},
\end{aligned}
$$

where $\hat{\psi}$ is a solution of

$$
\hat{\omega}(\rho, \theta, \tau)=-\left(\frac{\partial^{2} \hat{\psi}}{\partial \rho^{2}}+\frac{1}{\rho} \frac{\partial \hat{\psi}}{\partial \rho}+\frac{1}{\rho^{2}} \frac{\partial^{2} \hat{\psi}}{\partial \theta^{2}}\right) .
$$

The leading-order streamfunction is

$$
\begin{aligned}
& \hat{\psi}(\rho, \theta, \tau)=\hat{\psi}_{0}(\rho, \tau)+\sum_{-\infty, \neq 0}^{\infty}\left(\frac{f_{n}(\rho)}{n^{2} \tau^{2} \Lambda^{2}(\rho)}+O\left(\tau^{-3}\right)\right) \\
& \quad \times e^{i n[\theta-\Omega(\rho) \tau]} \\
& \Lambda(\rho)=\frac{d \Omega}{d \rho}
\end{aligned}
$$

where $\hat{\psi}_{0}(\rho, \tau)$ is the streamfunction corresponding to the evolution of the axisymmetric component of the spiral. This will be discussed separately later. The corresponding velocity components are

$$
\begin{aligned}
\hat{u}_{\rho}(\rho, \theta, \tau)= & \frac{1}{\rho} \sum_{-\infty, \neq 0}^{\infty}\left(\frac{i f_{n}(\rho)}{n \tau^{2} \Lambda^{2}(\rho)}+O\left(\tau^{-3}\right)\right) \\
& \times e^{i n[\theta-\Omega(\rho) \tau]}, \\
\hat{u}_{\theta}(\rho, \theta, \tau)= & \hat{u}_{\theta_{0}}(\rho, \tau)+\sum_{-\infty, \neq 0}^{\infty}\left(\frac{i f_{n}(\rho)}{n \tau \Lambda(\rho)}+O\left(\tau^{-2}\right)\right) \\
& \times e^{i n[\theta-\Omega(\rho) \tau]},
\end{aligned}
$$

where $u_{\theta_{0}}$ is the $n=0$ or axisymmetric component of the azimuthal velocity.

From (35) and (36), we then have, for the quadratic velocity products

$$
\begin{aligned}
& \hat{u}_{\theta}^{2}(\rho, \theta, \tau)=\hat{u}_{\theta_{0}}^{2}(\rho, \tau)+\sum_{-\infty, \neq 0}^{-\infty}\left(\frac{2 i \rho f_{n}(\rho) \Omega(\rho)}{n \tau \Lambda(\rho)}\right. \\
& \left.+O\left(\tau^{-2}\right)\right) e^{i n[\theta-\Omega(\rho) \tau]}, \\
& \hat{u}_{\rho} \hat{u}_{\theta}=O\left(\tau^{-2}\right), \quad \hat{u}_{\rho}^{2}=O\left(\tau^{-4}\right) .
\end{aligned}
$$

In squaring (36) to obtain (37) we have replaced $\hat{u}_{\theta_{0}}(\rho, \tau)$ by its initial value $\rho \Omega(\rho)$ when multiplied by the sum. This is justified since $\Omega(\rho, \tau)=\Omega(\rho, 0)+O\left(\tau^{-2}\right)$ in the evolution of the spiral: see Lundgren's Appendix A for details. 


\section{B. Pressure field}

Let $\hat{P}(\rho, \theta, \tau)$ be the pressure-density ratio for the equivalent two-dimensional flow. This can be obtained from the $\rho$-momentum equation for the inviscid vortex

$$
\frac{\partial \hat{u}_{\rho}}{\partial \tau}+\hat{u}_{\rho} \frac{\partial \hat{u}_{\rho}}{\partial \rho}+\frac{\hat{u}_{\theta}}{\rho} \frac{\partial \hat{u}_{\rho}}{\partial \theta}-\frac{\hat{u}_{\theta}^{2}}{\rho}=-\frac{\partial \hat{P}}{\partial \rho},
$$

by first expanding $\hat{P}$ in the form

$$
\begin{aligned}
\hat{P}(\rho, \theta, \tau)= & \hat{P}_{0}(\rho, \tau)+\sum_{-\infty, \neq 0}^{\infty}\left[\tau^{-1} \hat{P}_{n}^{(1)}(\rho)+\tau^{-2} \hat{P}_{n}^{(2)}(\rho)\right. \\
& \left.+\tau^{-3} \hat{P}_{n}^{(3)}(\rho)+\cdots\right] e^{i n[\theta-\Omega(\rho) \tau]}
\end{aligned}
$$

where $\hat{P}_{0}(\rho, \tau)$ is the $\theta$-averaged pressure field. Using (40) on the right side of (39) and (35)-(38) for the velocity components on the left-hand side we can obtain for the axisymmetric component, to leading order

$$
\frac{\hat{u}_{\theta_{0}}^{2}(\rho)}{\rho}=\frac{\partial \hat{P}_{0}}{\partial \rho} \text {. }
$$

For the nonaxisymmetric modes the $O\left(\tau^{0}\right)$ terms lead to $\hat{P}_{n}^{(1)}(\rho)=0$, and the $O\left(\tau^{-1}\right)$ terms give

$$
\hat{P}_{n}^{(2)}(\rho)=-\frac{2 \Omega(\rho) f_{n}(\rho)}{n^{2} \Lambda^{2}(\rho)} .
$$

Calculation of $\hat{P}_{n}^{(3)}$ requires inclusion of higher-order terms in inverse powers of $\tau$ in the expansion of the velocity components. The pressure field for the equivalent twodimensional vortex flow is then given by, to $O\left(\tau^{-2}\right)$

$$
\begin{aligned}
& \hat{P}(\rho, \theta, \tau)=\hat{P}_{0}(\rho, \tau)+\sum_{-\infty, \neq 0}^{\infty} \hat{P}_{n}(\rho, \tau) e^{i n \theta}, \\
& \hat{P}_{n}(\rho, \tau)=-\frac{2 \Omega(\rho) f_{n}(\rho)}{n^{2} \tau^{2} \Lambda^{2}(\rho)} e^{-i n \Omega(\rho) \tau} .
\end{aligned}
$$

The corresponding pressure produced by the stretched vortex is

$$
P(r, \theta, t)=e^{a t} \hat{P}(\rho, \theta, \tau),
$$

where the mapping from stretched variables $(r, t)$ to "twodimensional" variables $(\rho, \tau)$ is given by (28). Note that in (45) we have omitted, for reasons discussed in Sec. II, an additive term $-\frac{1}{2} a^{2}\left(\frac{1}{4} r^{2}+r_{3}^{2}\right)$ on the right-hand side which is the pressure from the irrotational strain field. We again put $l(t)=l_{0} \exp (a t), N=N_{c} l_{0} / L^{3}$ and rewrite (14) as

$$
\begin{aligned}
& \mathscr{T}(k)=\mathscr{G}_{0}(k)+\mathscr{F}_{s}(k), \\
& \mathscr{F}_{0}(k)=2 \pi k N \int_{\tau_{1}}^{\tau_{2}} I_{0} I_{0}^{*} d \tau, \\
& \mathscr{F}_{s}(k)=4 \pi k N \int_{\tau_{1}}^{\tau_{2}} \sum_{n=1}^{\infty} I_{n} I_{n}^{*} d \tau, \\
& I_{n}(k, \tau)=\int_{0}^{\infty} I_{n}\left(\frac{k \rho}{\sqrt{1+a \tau}}\right) \hat{P}_{n}(\rho, \tau) \rho d \rho,
\end{aligned}
$$

where $\mathscr{G}_{0}$ refers to that part of the spectrum which is produced by the $\theta$-averaged pressure and $\mathscr{G}_{s}$ arises from the nonaxisymmetry. Note that in the passage from (12) to (49), the integration over $r$ has been transformed to that over $\rho$ using (45) and (28).

\section{Nonaxisymmetric modes}

The integral $I_{0}$ from the axisymmetric field will be discussed separately later. When $k R$ is large, where $R$ is the cross-sectional scale of the vortex, the $I_{n}$ integrals, $n \neq 0$ can be estimated by the method of stationary phase with the result

$$
I_{n} I_{n}^{*} \approx \frac{\sqrt{1+a \tau}}{n \Lambda^{\prime}(\rho, \tau) \tau k} \rho\left|\hat{P}_{n}(\rho, \tau)\right|^{2},
$$

where it has been assumed that $\Lambda(\rho)<0, \Lambda^{\prime}=d \Lambda / d \rho>0$. In (50) $\rho(\tau, k, n)$ is to be interpreted as the point of stationary phase given by a solution of

$$
-k-n \sqrt{1+a \tau} \Lambda(\rho) \tau=0 .
$$

Substituting (50) and (44) into (48), and using (51) to transform the $\tau$ integral to one over $\rho$ then gives

$$
\mathscr{G}_{s}(k)=32 \pi k N \sum_{n=1}^{\infty} \int_{\rho_{1}}^{\infty} \frac{\rho f_{n}^{2}(\rho) \Omega^{2}(\rho)}{n^{5}|\Lambda(\rho)|^{5}} \frac{(1+a \tau)^{3 / 2}}{(2+3 a \tau) \tau^{4}} d \rho .
$$

In (52) we have put $\rho_{2}=\infty$ since the integrals may be shown to converge in this limit for the choice of $f_{n}$ to be discussed subsequently, but we have retained a finite early cutoff $\rho_{1}=\rho\left(\tau_{1}, k, n\right)$. The variable $\tau$ in (52) is of the form $\tau=\tau(\rho, k, n)$ from (51). When the approximation $(1+a \tau)^{1 / 2} \approx(a \tau)^{1 / 2}, a \tau \gg 1$ is made in (51) and (52), $\tau$ can be eliminated from (52) to give

$$
\mathscr{G}_{s}(k)=k^{-7 / 3} \frac{32 \pi N a^{5 / 3}}{3} \sum_{n=1}^{\infty} n^{-8 / 3} \int_{0}^{\infty} \frac{\rho f_{n}^{2}(\rho) \Omega^{2}(\rho)}{|\Lambda(\rho)|^{8 / 3}} d \rho,
$$

where we have now put $\rho_{1}=0$ with small error. The $k^{-7 / 3}$ dependence in (53) agrees with the Kolmogorov exponent of (4), but has been obtained from kinematical/dynamical rather than dimensional arguments. The present (53) is the analog for the pressure spectrum, of Eq. (88) of $\mathrm{L}$ for the energy spectrum. It is noteworthy that once again the stretched spiral-vortex model produces a spectral exponent that is independent of the detailed structure of the spiral.

\section{Some numerics}

For simplicity, and motivated by the mechanism of the roll-up of vortex sheets, we make the choice $f_{n}(\rho)=f(\rho)$, $n=0,1, \ldots$, in which case the spiral takes the form of a thin shear layer in the process of roll-up. We utilize a special choice of $f(\rho)$ and $g(\rho)$ by first introducing dimensionless forms of the functions $f(\rho), g(\rho), \Omega(\rho)$, and $\Lambda(\rho)$ as

$$
f(\rho)=\frac{\Gamma_{0}}{R^{2}} \tilde{f}(\xi), \quad g(\rho)=\frac{\Gamma_{0}}{R^{2}} \tilde{g}(\xi),
$$




$$
\Omega(\rho)=\frac{\Gamma_{0}}{R^{2}} \tilde{\Omega}(\xi), \quad \Lambda(\rho)=\frac{\Gamma_{0}}{R^{3}} \tilde{\Lambda}(\xi),
$$

where $\xi=\rho / R, \Gamma_{0}$ is a circulation scale, and $R$ is the initial lateral scale of the vortex structure. Note that the only independent functions are $\tilde{f}$ and $\tilde{g}$; the expressions for $\tilde{\Omega}(\xi)$, $\tilde{\Lambda}(\xi)$ in terms of $\tilde{f}$ and $\tilde{g}$ are the same as those connecting the equivalent dimensional quantities. Using (54) and (55) in (53), we may obtain

$\frac{\mathscr{G}_{s}(\tilde{k})}{\bar{\epsilon}^{3 / 4} \nu^{7 / 4}}=\mathscr{K}_{p} \tilde{k}^{-7 / 3}$,

$\mathscr{K}_{p}=\mathscr{E}\left(\frac{a^{2} \nu}{\bar{\epsilon}}\right)^{4 / 3}\left(\frac{\Gamma_{0}}{\nu}\right)^{4 / 3}\left(\frac{N R^{2}}{a}\right)$,

$\mathscr{C}=\int_{0}^{\infty} \mathscr{F}(\xi) d \xi \sum_{n=1}^{\infty} n^{-8 / 3}, \quad \mathscr{F}(\xi)=\frac{32 \pi}{3} \frac{\tilde{f}^{2} \tilde{\Omega}^{2} \xi}{|\tilde{\Lambda}|^{8 / 3}}$.

In the above $\tilde{k} \equiv k \eta$, where $\eta=\left(\nu^{3} / \bar{\epsilon}\right)^{1 / 4}$ is the Kolmogorov scale and $\bar{\epsilon}$ is the total dissipation. The quantity $\mathscr{C}$ is a pure number that can be calculated once $\tilde{f}$ and $\tilde{g}$ are specified, while the bracketed quantities in (57) are dimensionless parameters calculated by PS. Improved estimates are given by Pullin, Buntine, and Saffman ${ }^{18}$

Following PS we make a choice of $\tilde{f}(\xi), \tilde{g}(\xi)$ as

$$
\begin{aligned}
& \tilde{f}(\xi)=\frac{1}{\pi \Gamma[1+\alpha / 2]} \xi^{\alpha} e^{-\xi^{2}}, \\
& \tilde{g}(\xi)=-\frac{1}{\pi} \xi^{2} e^{-\xi^{2}} .
\end{aligned}
$$

In (59) $\alpha$ is a parameter with values in the range $0>\alpha \geqslant-1$. It is easily verified that $\tilde{\Lambda}<0$ as required. We make the choice ${ }^{15,18} \alpha=-1 / 2$. The integrand $\mathscr{F}$ can now be calculated as a function of $\xi$ and may be shown to be bounded at $\xi=0$ and integrable at $\xi \rightarrow \infty$ where exponential decay is found. The integral in (58) can then be calculated numerically and has the approximate value 0.4992 . Using $\Sigma n^{-8 / 3} \approx 1.28$ then gives $\mathscr{C} \approx 0.6390$.

Pullin, Buntine, and Saffman ${ }^{18}$ fix $\Gamma_{0} / \nu$ and calculate, with modeling assumptions, the remaining dimensionless parameters in (57) as functions of the Taylor-Reynolds number $R_{\lambda}$. These are reproduced in Table I which shows also the values of the prefactor $\mathscr{K}_{p}$. Shown for comparison are values of $1.3245 \mathscr{K}_{0}^{2}$, the Kolmogorov-Batchelor coefficient of $k^{-7 / 3}$ in (4).

\section{E. The axisymmetric component}

The axisymmetric or $\theta$-averaged velocity $\hat{u}_{\theta_{0}}$ is taken as a solution of the two-dimensional, radially-symmetric Navier-Stokes equations with initial conditions on the vorticity $\hat{\omega}_{0}(\rho, 0)=f(\rho)+g(\rho)$. This is

$$
\hat{u}_{\theta_{0}}(\rho, \tau)=\frac{1}{\rho} \int_{0}^{\rho} \hat{\omega}_{0}(s, \tau) s d s,
$$

TABLE I. Calculated values of $\mathscr{H}_{p}$ using values of $N R^{2} / a, a^{2} \nu / \epsilon$ from Ref. 18. $\mathscr{C}_{0}$ and $R_{\lambda}$ are their corresponding Kolmogorov prefactor and TaylorReynolds number, respectively. Top: $\Gamma_{0} / \nu=500$. Bottom: $\Gamma_{0} / \nu=1000$. The Kolmogorov-Batchelor pressure-spectrum prefactor is $1.325 \mathscr{K}_{0}^{2}$; see Eq. (4).

\begin{tabular}{rcccccr}
\hline \hline$\frac{a R^{2}}{4 \nu}$ & $\frac{N R^{2}}{a}$ & $\frac{a^{2} \nu}{\epsilon}$ & $\mathscr{K}_{0}$ & $\mathscr{C}_{p}$ & $1.325 \mathscr{K}_{n}^{2}$ & \multicolumn{1}{c}{$R_{\text {丸 }}$} \\
\hline 16 & 0.279 & 0.0129 & 2.14 & 2.14 & 6.07 & 45 \\
25 & 0.175 & 0.0198 & 1.75 & 2.37 & 4.06 & 215 \\
36 & 0.131 & 0.0258 & 1.60 & 2.52 & 3.39 & 732 \\
49 & 0.108 & 0.0310 & 1.48 & 2.66 & 2.90 & 2020 \\
64 & 0.093 & 0.0354 & 1.39 & 2.74 & 2.56 & 4814 \\
81 & 0.082 & 0.0393 & 1.33 & 2.77 & 2.35 & 10287 \\
100 & 0.075 & 0.0428 & 1.27 & 2.84 & 2.15 & 20194 \\
& & & & & & \\
16 & 0.607 & 0.0039 & 3.32 & 2.38 & 14.6 & 10 \\
25 & 0.284 & 0.0080 & 2.51 & 2.90 & 8.34 & 54 \\
36 & 0.191 & 0.0116 & 2.17 & 3.20 & 6.23 & 195 \\
49 & 0.147 & 0.0149 & 1.97 & 3.44 & 5.14 & 556 \\
64 & 0.122 & 0.0179 & 1.84 & 3.65 & 4.48 & 1354 \\
81 & 0.105 & 0.0204 & 1.74 & 3.73 & 4.01 & 2940 \\
100 & 0.093 & 0.0228 & 1.66 & 3.65 & 3.84 & 5843 \\
\hline \hline
\end{tabular}

$$
\begin{aligned}
\hat{\omega}_{0}(s, \tau)= & \frac{1}{2 \nu \tau} \exp \left(\frac{-s^{2}}{4 \nu \tau}\right) \int_{0}^{\infty}[f(u)+g(u)] \\
& \times \exp \left(-\frac{u^{2}}{4 \nu \tau}\right) I_{0}\left[\frac{s u}{2 \nu \tau}\right] u d u,
\end{aligned}
$$

where $I_{0}[\cdots]$ is the modified Bessel function of order zero. Substitution of (61) into (62), and the result into (16), and then (47) gives a multidimensional integral expression for $\mathscr{G}_{0}(k)$. Although (62) can be integrated in closed form for the initial conditions (59)-(60), evaluation of the remaining integrals for either general $\alpha$ or for the specific value $\alpha$ $=-1 / 2$ is not possible. Instead we make an estimate of the axisymmetric contribution by a calculation for one special value, $\alpha=0$, for which the integrals can be evaluated explicitly. This amounts to a change in one component of the initial conditions for the Navier-Stokes evolution of the vorticity, and we argue that the resulting axisymmetric pressure spectrum should be similar in form and in numerical magnitude to that which would result from a more complete calculation with $\alpha=-1 / 2$. The resulting contribution to $\mathscr{G}_{0}$ will be seen to be smaller than that produced by the nonaxisymmetric modes over inertial-range values of $k$.

With $\alpha=0$, the integrals (61) and (62) then give

$$
\hat{u}_{\theta_{0}}(\rho, \tau)=\frac{\Gamma_{0} R^{2} \rho}{2 \pi\left(R^{2}+4 \nu \tau\right)^{2}} \exp \left(-\frac{\rho^{2}}{\left(R^{2}+4 \nu \tau\right)^{2}}\right) .
$$

The integrals $I_{0}$ and $I_{2}$ are, from (16),

$$
\begin{aligned}
I_{0}= & \frac{\Gamma_{0}^{2} R^{4}\left[8(1+a \tau)-k^{2}\left(R^{2}+4 \nu \tau\right)\right]}{256 \pi^{2}(1+a \tau)\left(R^{2}+4 \nu \tau\right)^{2}} \\
& \times \exp \left(-\frac{k^{2}\left(R^{2}+4 \nu \tau\right)}{8(1+a \tau)}\right),
\end{aligned}
$$




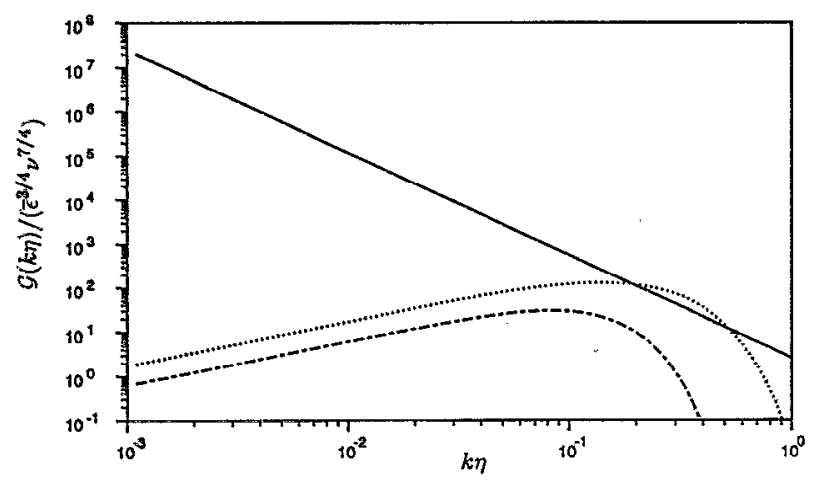

FIG. 2. Pressure spectra plotted as $\mathscr{G}(\tilde{k}) / \epsilon^{-3 / 4} \nu^{7 / 4}$ vs $k \eta$. Coefficients from Table I, $\Gamma_{d} \nu=500, a R^{2} / 4 \nu=30$. - present, Eq. (56). $\cdots$ axisymmetric mode, $\tau_{2}=\infty$. - - - ---axisymmetric mode with $\tau_{2}$ cutoff given by (66).

$$
I_{2}=\frac{\Gamma_{0}^{2} R^{4} k^{2}}{256 \pi^{2}(1+a \tau)\left(R^{2}+4 \nu \tau\right)} \exp \left(-\frac{k^{2}\left(R^{2}+4 \nu \tau\right)}{8(1+a \tau)}\right)
$$

respectively. When these expressions are used in (46) and (47), with the lower cutoff-time $\tau_{1}=0$, the $\tau$ integration can be performed giving lengthy expressions involving the exponential integral, which we do not reproduce here. The integrals were done in closed form using MATHEMATICA and each was checked by numerical evaluation for specific values of the parameters. The resulting axisymmetric pressure spectrum can be cast into a dimensionless form analogous to (56), as a function of $\tilde{k}$. We show numerical results for two choices of the upper cutoff $\tau_{2}$. The first is $\tau_{2}=\infty$, for which the $\tau$ integration converges owing to vorticity cancellation, and which then gives an upper limit for the axisymmetric spectrum. The second is the choice

$$
a \tau_{2}=C\left(\frac{R^{2} a}{4 \nu}\right)\left(\frac{\Gamma_{0}}{\nu}\right)^{-2 / 3}
$$

being the lifetime of the spiral; this value was used in Ref. 18 for the calculation of the quantities listed in Table I. The axisymmetric and nonaxisymmetric [from (56)] spectral components are shown in Figs. 2 and 3. To obtain these curves $\Gamma_{0} / \nu$ was fixed and $a R^{2} / 4 \nu$ was chosen to give a desired $R_{\lambda}$. The remaining dimcnsionless coefficients $N R^{2} / a$ and $a^{2} \nu / \vec{\epsilon}$ are then obtained from Table I. This is sufficient to

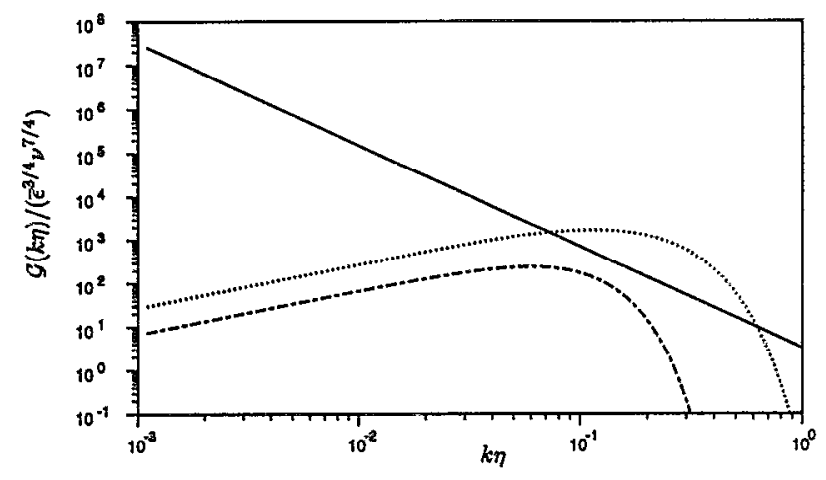

FIG. 3. Pressure spectra. $\Gamma_{0} / \nu=1000, a R^{2} / 4 \nu=36$. For key, see Fig. 2 .

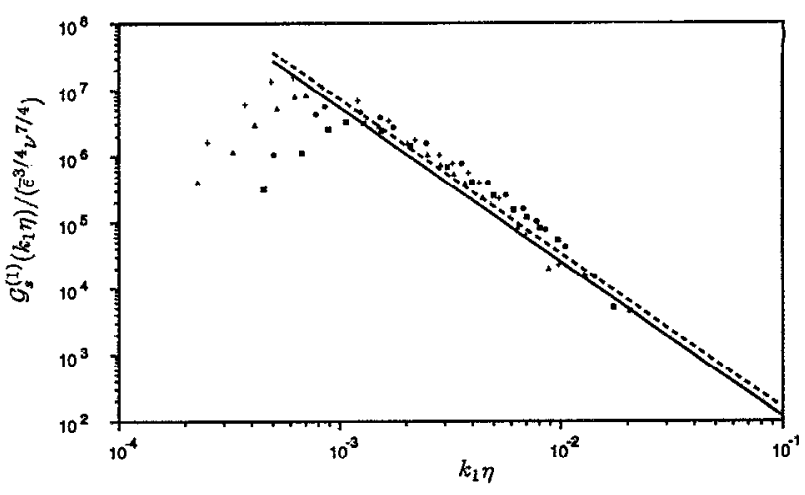

FIG. 4. One-dimensional pressure spectra plotted as $\mathscr{G}_{s}^{(1)}\left(k_{1} \eta\right) / \bar{\epsilon}^{-3 / 4} p^{7 / 4}$ vs $k_{1} \eta$. - present results, Eq. (56), $\Gamma_{0} / \nu=500, a R^{2} / 4 \nu=30$ (Table I). -..Kolmogorov-Batchelor. Symbols, George ${ }^{9}$ et al, $R_{\lambda} \approx 350-600$.

calculate all curves. No further parameters beyond those employed in Ref. 18 are required. In Figs. 2 and 3 it may be seen that the axisymmetric contribution calculated with (66) is always small compared to that produced by the nonaxisymmetric modes in the inertial range. For the energy (velocity) spectrum, the inertial range is usually taken to extend to $\tilde{k} \approx 1 / 8$, where the dissipation range begins. Assuming that this is also characteristic of the pressure spectrum, then Figs. 3 and 4 show that the $\tilde{k}^{-7 / 3}$ component of (56) appears to be dominant over the range of possible validity of the model. We recall that the present calculation, at least for the spiral modes, does not extend into the viscous range. The form of the axisymmetric component nonetheless suggests the possible presence of a bump in the pressure spectra at the beginning of the dissipation range $k>O(0.1)$.

\section{F. Comparison with measurement}

In Fig. 4 we compare the present results with the measurements of one-dimensional pressure spectra by George et $a l .{ }^{9}$ For isotropic turbulence the one-dimensional spectrum can be calculated from (56) from the relation

$$
\mathscr{G}_{s}^{(1)}\left(k_{1}\right)=\frac{1}{2} \int_{k_{1}}^{\infty} \frac{1}{\kappa} \mathscr{G}_{s}(\kappa) d \kappa,
$$

which gives

$$
\frac{\mathscr{F}_{s}^{(1)}\left(\tilde{k}_{1}\right)}{\bar{\epsilon}^{3 / 4} \nu^{7 / 4}}=\frac{3 \mathscr{K}_{p}}{14} \tilde{k}_{1}^{-7 / 3} .
$$

where $\tilde{k}_{1}=k_{1} \eta$ is the one-dimensional wave number. The data shown are taken from Fig. 15 of Ref. 9. The measurements were made in the mixing layer of a turbulent jet at 1.5-3 diameters downstream of the jet exit, using a condenser-microphone-type static pressure probe. Reynolds numbers based on the exit velocity and jet diameter were $4.0 \times 10^{5}$ and $6.2 \times 10^{5}$, and the Taylor-Reynolds numbers were in the range $R_{\lambda} \approx 350-600$. The spectra data has been converted from the published form, which was nondimensionalized with large-scale parameters, to the Kolmogorov scaling shown in Fig. 4 using estimates of the dissipation and of the Kolmogorov microscale given in Ref. 17 of Ref. 9. The authors give a lengthy discussion of possible sources of 
error and state that the limited validity of the Taylor's approximation used to convert from frequency to wave-number spectra together with velocity contamination caused by flowprobe interactions have had some effect on the accuracy of the data. The present result shown in Fig. 4 does not include the estimate of the axisymmetric contribution discussed in the previous section, and uses $\Gamma_{0} / \nu=500, a R^{2} / 4 \nu=30$, which, from Table I gives $R_{\lambda}$ in the range of the experiment; however use of $\Gamma_{0} / \nu=1000, a R^{2} / 4 \nu=49$, giving similar $R_{\lambda}$, changes the result only slightly. There is little difference between the theoretical curves over the range of the data, with which there is fair agreement. It would be interesting to compare the present results, and predictions of the KolmogorovBatchelor analysis with pressure spectra obtained from direct numerical simulation of isotropic turbulence.

\section{CONCLUDING REMARKS}

Our principal result is (53) which gives the shellsummed three-dimensional pressure spectrum for the nonaxisymmetric modes of the stretched spiral vortex. The $\tilde{k}^{-7 / 3}$ form can be traced to the product of the axisymmetric velocity with the $O\left(\tau^{-1}\right)$ component of the nonaxisymmetric field $u_{\theta}$ in the sum of (36). It is, therefore, a result of interaction between the radially symmetric and nonaxisymmetric spiral modes of the azimuthal velocity in the vortex. The present results show that it is possible to calculate spectra of higherorder quantities for stretched-vortex models of turbulent fine scales. In particular, the Lundgren stretched-spiral vortex again, as for the energy spectrum, produces an exponent in agreement with Kolmogorov-type dimensional analysis. The parameters used in Ref. 18 are sufficient for an estimate of the prefactor $\mathscr{K}_{p}$.

Support for the stretched spiral-vortex model in terms of visual sightings of nearly axisymmetric, coherent (in the sense of phase-amplitude correlation of the vorticity) structures with dimension of order the Taylor microscale from studies of numerical data bases of turbulent vorticity fields, is mixed. Vincent and Meneguzzi ${ }^{23}$ report vorticity in "...sheets, which tend to bend and roll up, producing the first vorticity tubes... ." In some cases "... a spiral structure due to the rolling up of a vorticity sheet..." is observed but this is a "...transient phase...." In our opinion the relevance of the Lundgren stretched spiral to a possibly universal vorticity structure of the fine scales remains an open question. Despite this we have a single model which appears able to produce plausible results for diverse properties of stationary isotropic turbulence such as the energy spectrum, ${ }^{11}$ the spectrum of passive and reacting scalars, ${ }^{12}$ the higher-order one-point statistics of the velocity and vorticity field, ${ }^{16}$ the enstrophy spectrum ${ }^{18}$ and the spectra of higher-order quantities such as the pressure. If stretched spiral-like structures, or some close relative are found not to be characteristic of the fine scales of turbulence, there remains the nontrivial puzzle of why the model is successful in reproducing these experimentally and numerically observed properties? Perhaps a more general type of structure is indicated. An alternative is that generic model features such as kinematical self-consistency combined with an explicit mechanism for inertial transfer based on hard contact with Navier-Stokes dynamics inclusive of vortex stretching are sufficient to capture the essential physics of the upper inertial and dissipation-range properties of stationary turbulence. The specific details of the vorticity field in the sense of some preferred structure may then be of secondary importance.

\section{ACKNOWLEDGMENTS}

The author wishes to thank P. G. Saffman for many useful discussions and for his comments on the original manuscript. Partial support under NSF Grant No. CTS-9311811 is gratefully acknowledged.

${ }^{1}$ W. Heisenberg, "Zur statischen Theorie der Turbulenz," Z. Phys. 124, 628 (1948).

"S. Chandrasekhar, "On Heisenberg's elementary theory of turbulence," Proc. R. Soc. London Ser. A 200, 20 (1949).

${ }^{3}$ A. M. Obukhov, "Pressure fluctuations in a turbulent flow," Dokl. Akad. Nauk SSSR Ser. Geofiz. 3, 49 (1949).

${ }^{4}$ A. M. Yaglom, "Acceleration in a turbulent flow," Dokl. Akad. Nauk SSSR 67, 795 (1949).

${ }^{5}$ A. S. Monin and A. M. Yaglom, Statistical Fluid Mechanics (MIT Press, Cambridge, MA, 1949), Vol. II.

${ }^{6} \mathrm{G}$. K. Batchelor, "Pressure fluctuations in isotropic turbulence," Proc. Cambridge, Philos. Soc. 47, 359 (1951).

${ }^{7}$ G. K. Batchelor, The Theory of Homogeneous Turbulence (Cambridge University Press, Cambridge, 1953).

${ }^{8} \mathrm{R}$. H. Kraichnan, "Pressure field within homogeneous isotropic turbulence," J. Acoust. Soc. Am. 28, 64 (1956).

${ }^{9}$ W. K. George, P. D. Beuther, and R. E. A. Arndt, "Pressure spectra in turbulent free shear flows," J. Fluid Mech. 148, 155 (1984).

${ }^{11}$ M. D. Millionshchikov, "Theory of homogeneous isotropic turbulence," Izv. Akad. Nauk. SSSR Ser. Geofiz. 5, 433 (1941).

${ }^{11}$ T. S. Lundgren, "Strained spiral vortex model for turbulent fine structure," Phys. Fluids 25, 2193 (1982).

${ }^{12} \mathrm{~T}$. S. Lundgren, "The concentration spectrum of the product of a fast biomolecular reaction," Chem. Eng. Sci. 40, 1641 (1985).

${ }^{13}$ T. S. Lundgren, “A small scale turbulence model," Phys. Fluids A 5, 1472 (1993).

${ }^{14}$ A. A. Townsend, "On the fine-scale structure of turbulence," Proc. R. Soc. London Ser. A 208, 534 (1951).

${ }^{15} \mathrm{~J}$. M. Burgers, "A mathematical model illustrating the theory of turbulence," Adv. Appl. Mech. 1, 171 (1948).

${ }^{16}$ D. I. Pullin and P. G. Saffman, "On the Lundgren-Townsend model of turbulent fine scales," Phys. Fluids A 5, 126 (1993).

${ }^{17}$ P. G. Saffman and D. I. Pullin, "Anisotropy of the Lundgren-Townsend model of fine-scale turbulence," Phys. Fluids 6, 802 (1994).

${ }^{18}$ D. I. Pullin, J. D. Buntine, and P. G. Saffman, "On the spectrum of a stretched spiral vortex," Phys. Fluids 6, 3010 (1994).

${ }^{19} \mathrm{R}$. M. Kerr, "Higher-order derivative correlations and the alignment of small-scale structures in isotropic numerical turbulence," J. Fluid Mech. 153, 31 (1985). See, also, NASA TM No. 84407, 1983.

${ }^{20}$ W. T. Ashurst, A. R. Kerstein, R. M. Kerr, and C. H. Gibson, "Alignment of vorticity and scalar-gradient in simulated Navier-Stokes turbulence," Phys. Fluids 30, 2343 (1987).

${ }^{21} \mathrm{~A}$. Vincent and M. Meneguzzi, "The spatial structure and statistical properties of homogeneous turbulence," J. Fluid Mech. 225, 1 (1991).

${ }^{22}$ A. Abramowitz and I. S. Stegun, Handbook of Mathematical Functions (Dover, New York, 1965).

${ }^{23}$ A. Vincent and M. Meneguzzi, "The dynamics of vorticity tubcs in homogeneous turbulence," J. Fluid Mech. 258, 245 (1994). 